\title{
Transitioning Semiconductor Companies Enabling Smart Environments and Integrated Ecosystems
}

\author{
Samar K. Saha \\ Prospicient Devices, Milpitas, CA, USA \\ Email:samar@ieee.org
}

How to cite this paper: Saha, S.K. (2018) Transitioning Semiconductor Companies Enabling Smart Environments and Integrated Ecosystems. Open Journal of Business and Management, 6, 428-437. https://doi.org/10.4236/ojbm.2018.62031

Received: March 27, 2018

Accepted: April 25, 2018

Published: April 28, 2018

Copyright $\odot 2018$ by author and Scientific Research Publishing Inc. This work is licensed under the Creative Commons Attribution International License (CC BY 4.0).

http://creativecommons.org/licenses/by/4.0/ (c) (1) Open Access

\begin{abstract}
This paper discusses the business opportunities to the semiconductor companies transitioning from "pure-play" compute-focused chip providers only to complete solution providers enabling smart environments and integrated ecosystems. The semiconductor industry has led to the foundation of the Internet and Information age. The Internet enables connecting smart devices or "things" to be operated without human interactions creating Internet of Things (IoT). The IoT-enabled smart devices are being integrated into a single ecosystem with a shared user interface for healthcare, security, energy saving, automation, autonomous vehicles, telecommunications, computers, entertainment, and so on. Thus, the IoT offers semiconductor companies great business opportunities to provide IoT-enabling components for interoperability as well as system integration and software support. In this paper, the key IoT-enabling technologies and role of semiconductor companies transitioning from component suppliers only to complete IoT solution providers enabling smart environments and integrated ecosystems are discussed.
\end{abstract}

\section{Keywords}

Internet of Things, Smart Homes, Smart Cars, Smart Cities, Smart Health, Smart Environment, Smart Things, Cloud Computing, Sensors, Connectivity, Computing, Semiconductor Industry, Transitioning Business Model

\section{Introduction}

The semiconductor industry continues to have an unprecedented impact on improving almost every aspect of modern society including communications, military, security, healthcare, energy saving, industrial automation, transport, and 
entertainment [1] [2]. Over the last four decades, the relentless pursuit of integrated circuit (IC) device miniaturization for manufacturing high-performance and high-density IC-chips and system-on-a-chip (SoC) led to the creation of Internet and social media [2]-[7]. The semiconductor components are used in smart cars, smart homes, smart cities, smart health, smart energy, smart security, smart appliances, and so on [8]. The Internet enables to connect any and every smart devices or "things" creating "Internet of Things" (IoT) [9] [10] and Internet of everything (IoE) [11]. And, the IoT-connected smart devices constitute a smart environment and integrated ecosystem that can be accessed via personal computers, tablets, and smartphones from anywhere without human interaction [12] [13].

The IoT enables smart environments or spaces and self-aware things including smart transport, energy, rural areas, buildings or homes, cities, health, and so on for easy access to mobility, climate, food, living, digital society, and health care applications [12] [13] [14] [15]. The IoT is growing tremendously worldwide and the estimated business opportunities for the semiconductor industry from the IoT expansion will exceed US\$4 trillion worldwide in 2025 [16]. The IoT-enabling semiconductor products that continue to post revenue growth include smart thermostats, wearable fitness devices, and other innovations [16]. And, the IoT-enabling semiconductor components ranging from medical-monitoring systems to sensors for autonomous vehicles are expected to offer even greater growth [16]. The forecasted global semiconductor market growth in 2019 for data processing segment is the largest with a total of US $\$ 162$ billion, followed by the communications segment with a total of US $\$ 127$ billion whereas, the strongest forecasted growth is in the industrial segment with a compound annual growth rate (CAGR) of 9.7 percent and automotive segment with a CAGR of 8.2 percent [17].

The estimated growth in revenue, in trillions of US dollars reflects the transformational potential of IoT in both consumer and business-to-business applications [16] [17] [18]. The semiconductor companies providing IoT-enabling hardware, software, and integration services will benefit the most from IoT expansion. Therefore, it is vast business opportunities for transitioning semiconductor companies from "pure-play" component suppliers only to complete IoT solution providers by offering IoT-enabling products along with hardware and software support. However, since the volume of IoT-enabling niche products is relatively low, some companies choose business as usual as component suppliers instead of capital investment for developing IoT-enabling niche products. In this article, the opportunities and role of semiconductor companies as complete IoT solution providers enabling smart environments and integrated ecosystems is presented.

The objective of this study is to present the opportunities and role of semiconductor companies transitioning to complete solution providers enabling smart environments and integrated ecosystems. In order to achieve this objec- 
tive, first of all, a brief overview of IoT is presented followed by discussions on the IoT-enabling key technologies. Then the role of semiconductor companies as the IoT-enablers along with the transitioning business model for complete IoT solution providers is presented. Finally, the conclusion of the study on transitioning semiconductor companies from pure-play component suppliers only to complete solution providers enabling smart environments and integrated ecosystems is presented.

\section{Enablers for Smart Environments and Integrated Ecosystems}

In order to discuss the business opportunities for semiconductor companies transitioning from pure-play compute-focused IC-chip suppliers only to complete solution providers enabling smart environments and integrated ecosystems, let us present a brief overview of IoT and key IoT-enabling technologies.

\subsection{The Internet of Things (IoT)}

The ubiquitous Internet enables connecting smart things or devices such as autonomous vehicles, homes, appliances, cities, healthcare facilities, and so on to form an integrated network [10] [11]. Here, smart things represent any product, or system, or individual network with sensors and actuators that can be accessed through the Internet from any computing environment and via cloud computing. This interconnected things or network of networks is known as the IoT [10] [11]. Thus, the IoT can be defined as a network containing all smart devices or things with some sort of sensing mechanism that can communicate via the Internet with other smart devices or the cloud without human interaction [12]. In other words, the IoT is a network consisting of any self-aware object that can be accessed through the Internet using personal computers, tablets, and smartphones as shown in Figure 1.

Figure 1(a) shows individual networks connected to form network of networks whereas, Figure 1(b) shows IoT ecosystem with networking between people-to-people ( $\mathrm{P} 2 \mathrm{P})$, people-to-machine ( $\mathrm{P} 2 \mathrm{M})$, machine-to-machine (M2M), and people-to-business (P2B). Thus, the IoT infrastructure offers P2P, P2M, and $\mathrm{M} 2 \mathrm{M}$ communications enabling appliances and services providing notifications, security, energy saving, automation, telecommunications, healthcare, computers, entertainment, digital society, and so on to create a smart environment with a shared user interface. This ongoing progress of smart environments and integrated ecosystems is enabled by semiconductor industry's continuous miniaturization of IC devices providing low-cost, high-density, fast, low-power, and high-yielding semiconductor components for IoT infrastructure [19] [20] [21] [22] [23].

With the worldwide growth of IoT, the semiconductor companies have a great opportunity to transition from component suppliers only to complete IoT solution providers. In this respect, the semiconductor companies must identify the 


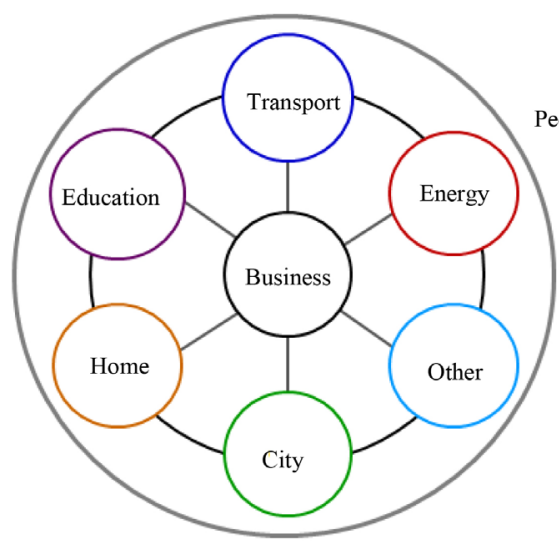

(a)

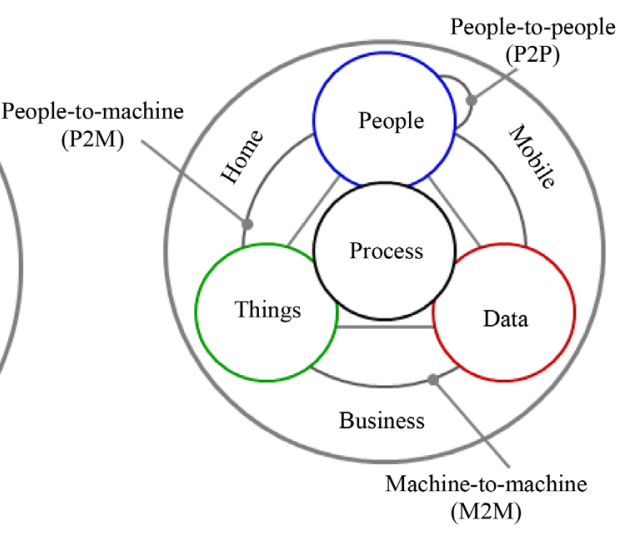

(b)

Figure 1. The IoT ecosystem: (a) Individual networks are connected to each other via the Internet forming network of networks [10]; (b) IoT process enabling people-to-people, people-to-machine, machine-to-machine communications [11]. In Figure, business, transport, energy, education, home, city, and things represent individual networks; whereas, "things" represent smart devices that can be operated by any computing device via the Internet.

key IoT-enabling technologies to manufacture IoT-enabling components and provide software and hardware support to IoT infrastructure developers. In the following Subsection 2.2, the key IoT-enabling technologies are described.

\subsection{Key IoT-Enabling Technologies}

The IoT-enabling semiconductor technologies include the state-of-the-art manufacturing processes for high-performance IC-chips and SoCs to provide smart-electronic devices including self-driving cars, planes that fly automatically in a controlled space, smart homes, smart-city infrastructure, smart wearable fitness devices, and smart healthcare facilities. The key enabling IC manufacturing and Internet technologies for the existing and future networking infrastructure include [12]:

- Advanced IC manufacturing technologies;

- Micro and nano electronics;

- Smart materials;

- Photonics;

- Biotechnology;

- Internet Protocol (IP) addresses enabling a global and ubiquitous addressing of any communicating smart thing.

Using the above key enabling technologies, the IoT creates smart networking environments in real time either at physical or at cyber-space level.

With respect to key IC manufacturing technology, the semiconductor companies develop a new generation IC fabrication technologies in about every two years by aggressively scaling down [3] devices to improve IC performance along with reduced power at a reduced cost as predicted by Moore's Law [2]. The International Technology Roadmap for Semiconductors guided the "miniaturiza- 
tion" specifications and device performances [24]. Though the Moore's Law has slowed down in nanometer node generation, the trend for increased performances continues by power-performance trade-offs based on the application specific ICs, incorporation of smart materials in device fabrication, and the adoption of new transistor concepts [25] [26] [27]. This process of scaling is referred to as the "More Moore" [24] [28]. Another approach of increasing performance is the functional diversification of semiconductor devices in IoT-enabling IC-chips. This process of added functionality is known as the "More-than-Moore" [28].

In addition to IC manufacturing technology, the wireless sensor networks, 2G/3G/4G/5G data technology for cellular networks, RFID (radio-frequency identification), and so on are considered as the IoT-enabling technologies. The integrated networking, information processing, and sensing and actuation capabilities allow physical devices to operate in changing environments. These tightly coupled cyber and physical systems, referred to as the "cyber-physical" systems, exhibit high level of integrated intelligence and are part of the IoT-enabling technologies where the computational and physical processes of such systems are tightly interconnected and coordinated to work together effectively, with or without the humans in the loop. Thus, the role of semiconductor companies is critical in building the IoT ecosystem as described in Section 3.

\section{The Role of Semiconductor Companies as Enablers of Smart Environments and Integrated Ecosystems}

The worldwide expansion of IoT offers semiconductor companies huge business opportunities in the fastest-growing wireless communications market segment to gain leadership position in networking equipment and industrial automation. Since all IoT-enabling new devices and services require additional cloud infrastructure, IoT, also, offers a growth potential for networking ICs and servers for data centers. Thus, the IoT would significantly boost semiconductor revenues by stimulating demands for microcontrollers, sensors, connectivity, and memory chips and continue to maintain the average annual revenue increase of 3 to 4 percent overcoming the slower growth in the smartphone market [16].

In addition to triggering demands for IoT-enabling new semiconductor components and services, the IoT would increase the need for legacy products [16]. Thus, instead of capital investment for IoT-enabling niche product line, some semiconductor companies may repurpose their existing products as IoT-enablers such as repurposed wireless processors and chip sets by wireless SoC manufacturers and repurposed lower-end processors and connectivity chip-sets by microcontroller companies. With the tremendous growth potential in the global IoT market, the conservative business strategy for semiconductor companies is to aggressively pursuing the most growing application segments of the market [16] including:

- Wearable devices for smart health such as fitness monitoring accessories;

- Medical electronics; 
- Smart-home applications like automatic lighting and heating, security, and self-aware appliances enabling M2M communications;

- Industrial automation including tasks such as remote servicing and predictive maintenance;

- Autonomous vehicles;

- Smart-city infrastructure including applications to assisting traffic control and other tasks within the public sector enabling P2M and M2M communications.

In the IoT ecosystem, the semiconductor companies provide the technology building blocks for the self-aware things to help collecting, processing, and transmitting real world signals and data for IoT products and applications [17] [18]. The core capabilities and essentials for the IoT infrastructure, 1) sensing, 2) connectivity, and 3) computing, offer a huge IoT-market growth and great business opportunities for the semiconductor companies. The market growth for sensing in IoT applications is estimated to be the fastest, followed by computing and connectivity. However, the computing is expected to be more than 50 percent of the total IoT-enabling semiconductor revenues whereas, the remainder of the revenues is expected to split almost equally between the sensing and connectivity IC-chips [17]. The core capabilities for IoT infrastructure are discussed below.

\subsection{Sensing}

A sensor is an important component of any self-aware thing including smartphones, tablets, wearables, healthcare, and automotive in enabling smart environments and integrated ecosystems. The rapid growth of sensor market offers business opportunities to semiconductor companies to develop and supply sensors by combining electronics and mechanical components at micro and nano scales of advanced micro-electro-mechanical systems (MEMS) and nano-electro-mechanical systems (NEMS) manufacturing technologies. For variety of IoT applications, an integrated chip is required by integrating the functionalities of sensors, actuators, and ICs into small form-factors [17].

\subsection{Connectivity}

In IoT applications, the connectivity is a key capability required for consumer as well as enterprise infrastructure devices. Therefore, connectivity capabilities must be integrated in IoT-enabling devices and applications. The estimated growth in overall communications electronics market for semiconductors at a rate of 4.8 percent will reach US $\$ 127$ billion by 2019 [17]. The fastest growing smartphone device sector causing explosion in mobile data traffic is driving the growth in the mobile infrastructure equipment.

For IoT applications beyond smartphones, the connectivity and network processing-related functions are integrated with embedded processors. Furthermore, the "combo wireless" connectivity chipsets with integrated NFC 
(near-field communication), $\mathrm{Wi}-\mathrm{Fi}$, and Bluetooth are expected to grow strongly in smartphones and consumer electronic devices that are expected to support multiple communication standards and protocols. This trend in connectivity market segment is clearly a business challenge to pure-play compute-focused chip providers that do not manufacture products with a robust connectivity solution for IoT infrastructure

\subsection{Computing}

The IoT expansion will drive higher growth in application-specific microcontroller units (MCUs) with connectivity and flexible SoC designs to meet the demands for higher computing requirements of sophisticated real-time sensors for automotive, industrial, and medical applications. For example, Qualcomm's Snapdragon $602 \mathrm{~A}$ is an integrated chipset solution that supports advanced smartphone-level multimedia, navigation, connectivity, and voice quality and control features in cars [29]. Also, the growth of application specific MCUs that have integrated connectivity allows many home appliances and industrial devices to connect to the internet enabling smart homes integrated to the IoT ecosystem.

Again, the IoT applications require a single chip at an acceptable form-factor with very low power consumption suitable for battery operated devices. These requirements have driven the growth in the number of SoC-type designs comprising multiple embedded cores, embedded GPU (graphics processing units), and integrated wireless connectivity in a single package. The heterogeneous integration roadmap for electronics packaging will address the hardware challenges to integrate multiple functionalities in a single package in response to IoT driving forces of data centers, smartphones, and intelligent automotive to enabling smart environments and integrated ecosystems [30].

\subsection{Business Strategy for Semiconductor Companies}

With the huge growth potential of IoT worldwide, the semiconductor companies can take leadership position by developing advanced technologies, IoT-enabling products, and IoT integration services. Figure 2 shows the business model for transitioning semiconductor companies in enabling smart environments and integrated ecosystems.

Figure 2 shows that the semiconductor companies must adopt a suitable option to take advantage of vast business opportunities offered by expansion of IoT. In option 1, a company may repurpose its existing legacy products to IoT-enabling components instead of capital investment to develop niche products with a relatively low sales volume. In this option, the companies with existing wireless SoC product line may offer repurposed wireless processors and chip sets for IoT whereas, the companies with microcontroller products may bundle lower-end processors and connectivity chip-sets to compete for the same business. In second option, a semiconductor company may aggressively pursue to 


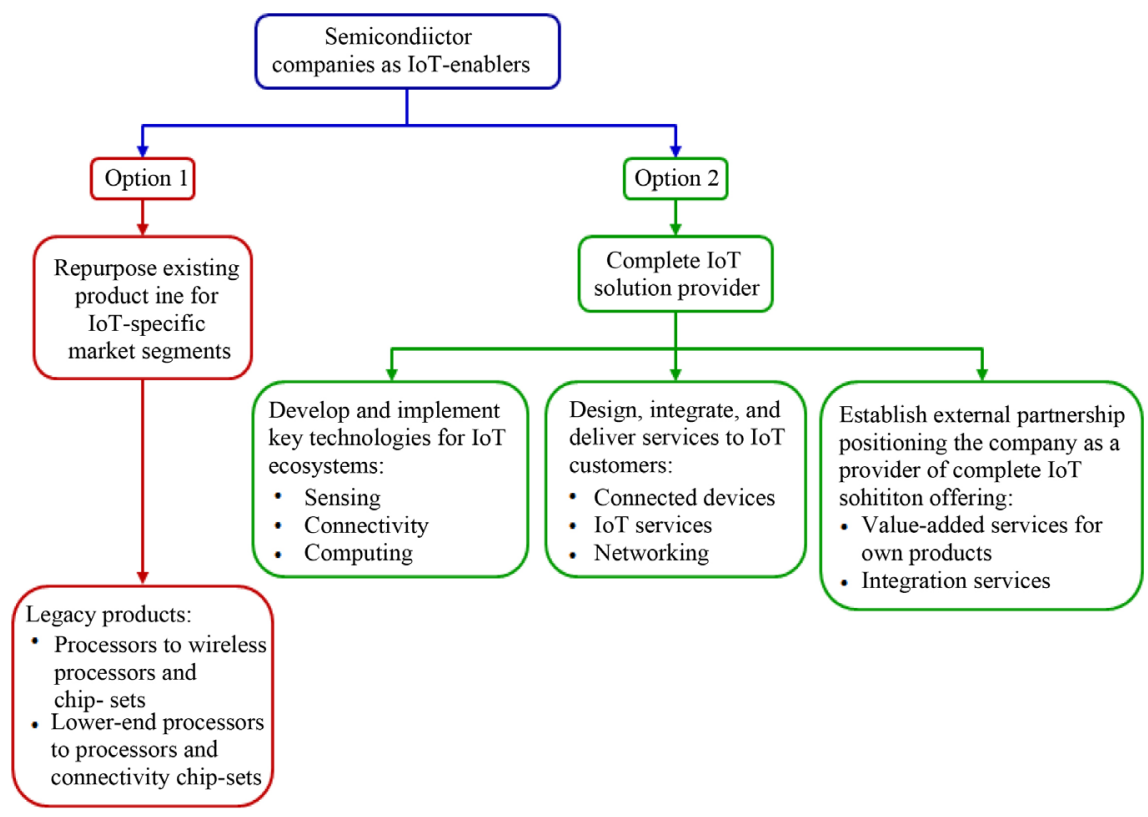

Figure 2. The role of semiconductor companies transitioning to complete IoT solution providers enabling smart environments and integrated ecosystems.

provide complete solution for IoT by developing key IoT-enabling technologies, core products including sensors, interoperability SoCs, and computing chips along with value-added services for implementation and integration of IoT infrastructure.

\section{Conclusions}

This study shows that the IoT will drive the rapid growth in the semiconductor industry maintaining the percentage growth rate in the revenue of the past decade in spite of the levelling off revenue in the smartphone business. Thus, the semiconductor companies must take advantage of this opportunity to develop or repurpose IoT-enabling technologies, products, and provide IoT-enabling services for hardware, software, and integration.

Though the IoT-enabling IC markets such as wearables, smart televisions, and autonomous vehicles offer tremendous business opportunities to semiconductor companies, there are many unresolved challenges including privacy and security. The semiconductor companies as enablers of the IoT ecosystem will play a critical role in providing the basic building blocks required to developing innovative solutions. In providing the appropriate solutions to the existing IoT challenges, the semiconductor companies must carefully consider to capture value from the IoT and gain leadership position. Thus, the semiconductor companies must develop a comprehensive strategic plan to engage in the profitable segments of the IoT market along with a solid execution plan exploring paths to close portfolio gaps and subsequently engaging with partners across the entire IoT value chain. The strategic planning for IoT engagement and solid execution plan will be crucial for the semiconductor companies transitioning from pure-play com- 
pute-focus IC-chips providers to complete IoT solution providers enabling smart environments and integrated ecosystems.

\section{References}

[1] Saha, S.K. (2016) Compact Models for Integrated Circuit Design: Conventional Transistors and Beyond. CRC Press, Taylor and Francis Group, Boca Raton, Florida.

[2] Moore, G.E. (1965) Cramming More Components onto Integrated Circuits. Electronics, 38, 114-117.

[3] Dennard, R.H., Gaensslen, F.H, Yu, H.N, Rideout, V.L., Bassous, E. and LeBlanc, A.R. (1974) Design of Ion-Implanted MOSFETs with Very Small Physical Dimensions. IEEE Journal of Solid State Circuits, 9, 256-268. https://doi.org/10.1109/JSSC.1974.1050511

[4] Iwai, H. (1999) CMOS Technology-Year 2010 and beyond. IEEE Journal of Solid State Circuits, 34, 357-366. https://doi.org/10.1109/4.748187

[5] Saha, S. (2001) Scaling Considerations for High Performance 25 nm Metal-Oxide-Semiconductor Field-Effect Transistors. Journal of Vacuum Science \& Technology B, 19, 2240-2246. https://doi.org/10.1116/1.1420207

[6] Saha, S.K. (2002) Transistors Having Optimized Source-Drain Structures and Methods for Making the Same. US Patent No. 6344405.

[7] Kuhn, K.J. (2012) Considerations for Ultimate CMOS Scaling. IEEE Trans. Electron Devices, 59, 1813-1828. https://doi.org/10.1109/TED.2012.2193129

[8] Saha, S.K. (2016) Emerging Business Trends in the Microelectronics Industry. Open Journal of Business and Management, 4, 105-113. https://doi.org/10.4236/ojbm.2016.41012

[9] Madakam, S., Ramaswamy, R. and Tripathi, S. (2015) Internet of Things: A Literature Review. Journal of Computer and Communications, 3, 164-173. https://doi.org/10.4236/jcc.2015.35021

[10] Evans, D. (2011) The Internet of Things-How the Next Evolution of the Internet Is Changing Everything. CISCO White Paper. http://www.cisco.com/web/about/ac79/docs/innov/IoT_IBSG_0411FINAL.pdf

[11] Evans, D. (2012) The Internet of Everything: How More Relevant and Valuable Connections Will Change the World. https://www.cisco.com/c/dam/global/en_my/assets/ciscoinnovate/pdfs/IoE.pdf

[12] Vermesan, O. and Friess, P., Eds. (2013) Internet of Things-Converging Technologies for Smart Environments and Integrated Ecosystems. River Publishers, Denmark.

[13] Vermesan, O. and Friess, P., Eds. (2011) Internet of Things-Global Technological and Societal Trends: Environments and Spaces to Green ICT. River Publishers, Denmark.

[14] Islam, S.M.R., Kwak, D., Kabir, M.H, Hossain, M. and Kwak, K.-S. (2015) The Internet of Things for Health Care: A Comprehensive Survey. IEEE Access, 3, 678-708. https://doi.org/10.1109/ACCESS.2015.2437951

[15] Zanella, A., Bui, N., Castellani, A., Vangelista, L. and Zorzi, M. (2014) Internet of Things for Smart Cities. IEEE Internet of Things Journal, 1, 22-32. https://doi.org/10.1109/JIOT.2014.2306328

[16] Bauer, H., Patel, M. and Veira, J. (2015) Internet of Things: Opportunities and 
Challenges for Semiconductor Companies.

https://www.mckinsey.com/industries/semiconductors/our-insights/internet-of-thi ngs-opportunities-and-challenges-for-semiconductor-companies

[17] Chitkara, R., Ballhaus, W., Acker, O., Song, B., Sundaram, A. and Popova, M. (2015) The Internet of Things: The Next Growth Engine for the Semiconductor Industry.

https://www.pwc.de/de/technologie-medien-und-telekommunikation/assets/pwc-st udie-prognostiziert-boom-in-der-halbleiterbranche.pdf

[18] Gloger, M., Mehrotra, R., Ogrins, A. and Sundaram, A. (2017) Strategy \& Semiconductor Industry \& Strategies for Growth in the Internet of Things Era. http://www2.caict.ac.cn/zscp/qqzkgz/qqzkgz_zdzsq/201706/P0201706203942021687 $\underline{42 . p d f}$

[19] Saha, S. (1998) Effects of Inversion Layer Quantization on Channel Profile Engineering for nMOSFETs with $0.1 \mu \mathrm{m}$ Channel Lengths. Solid-State Electronics, 42, 1985-1991. https://doi.org/10.1016/S0038-1101(98)00183-X

[20] Saha, S. (2001) Design Considerations for $25 \mathrm{~nm}$ MOSFET Devices. Solid-State Electronics, 45, 1851-1857. https://doi.org/10.1016/S0038-1101(01)00197-6

[21] Saha, S.K. (2007) Design Considerations for Sub-90-nm Split-Gate Flash Memory Cells. IEEE Transactions on Electron Devices, 54, 3049-3055. https://doi.org/10.1109/TED.2007.907265

[22] Colinge, J.-P., Ed. (2008) FinFETs and Other Multi-Gate Transistors. Springer, New York. https://doi.org/10.1007/978-0-387-71752-4

[23] Saha, S.K. (2014) Compact MOSFET Modeling for Process Variability-Aware VLSI Circuit Design. IEEE Access, 2, 104-115. https://doi.org/10.1109/ACCESS.2014.2304568

[24] (2015) International Technology Roadmap for Semiconductors: More Moore. https://www.semiconductors.org/clientuploads/Research_Technology/ITRS/2015/5 2015\%20ITRS\%202.0_More\%20Moore.pdf

[25] Saha, S.K. (2016) Transistor Structure and Method with an Epitaxial Layer over Multiple Halo Implants. US Patent No. 9299702.

[26] Saha, S.K. (2017) Transistor Structure and Fabrication Methods with an Epitaxial Layer over Multiple Halo Implants. US Patent No. 9768074.

[27] Liu, T.-J.K. and Kuhn, K., Eds. (2015) CMOS and Beyond: Logic Switches for Terascale Integrated Circuits. Cambridge University Press, Cambridge, UK. https://doi.org/10.1017/CBO9781107337886

[28] Arden, W., Brillouët, M., Cogez, P., Graef, M., Huizing, B. and Mahnkopf, R. (2010) "More than Moore" White Paper. http://www.itrs2.net/uploads/4/9/7/7/49775221/irc-itrs-mtm-v2_3.pdf

[29] QUALCOMM. https://www.qualcomm.com/products/snapdragon/processors/602a

[30] Chen, W. and Bottoms, W.R. (2017) Heterogeneous Integration Roadmap. 2017 IEEE International Conference on Electronics Packaging (ICEP), Yamagata, 19-22 April 2017, 302-305. https://doi.org/10.23919/ICEP.2017.7939380 